\title{
Electronic structure and bulk properties of $\mathrm{MB}_{6}$ and $\mathrm{MB}_{12}$ borides
}

\author{
G.E. Grechnev, A.E. Baranovskiy, V.D. Fil, T.V. Ignatova, \\ I.G. Kolobov, and A.V. Logosha \\ B. Verkin Institute for Low Temperature Physics and Engineering of the National Academy of Sciences of Ukraine \\ 47 Lenin Ave., Kharkov 61103, Ukraine \\ E-mail: grechnev@ilt.kharkov.ua
}

N.Yu. Shitsevalova and V.B. Filippov

Institute for Problems of Materials Science of the National Academy of Sciences of Ukraine 3 Krzhyzhanovsky Str., Kiev 03680, Ukraine

\section{Olle Eriksson}

Department of Physics, University of Uppsala, Box 530, SE-751 21 Uppsala, Sweden

Received May 21, 2008

\begin{abstract}
$A b$ initio band structure calculations have been carried out for higher borides $\mathrm{MB}_{6}$ and $\mathrm{MB}_{12}$. High precision measurements of the elastic constants were performed for $\mathrm{ZrB}_{12}, \mathrm{HoB}_{12}, \mathrm{ErB}_{12}, \mathrm{TmB}_{12}, \mathrm{LuB}_{12}, \mathrm{YB}_{6}$ and $\mathrm{LaB}_{6}$ compounds at low temperatures. The bulk properties of the borides have been analyzed on the basis of the calculated equations of states and balanced crystal orbital overlap populations. Our calculations indicate that hexaborides with divalent metals, $\mathrm{CaB}_{6}, \mathrm{SrB}_{6}, \mathrm{BaB}_{6}$, and $\mathrm{YbB}_{6}$, are semiconductors with small energy gaps. The metallic $\mathrm{MB}_{6}$ hexaborides with trivalent $\mathrm{M}$ atoms are found to possess larger bulk moduli values. For dodecaborides bulk moduli are found to be higher for $\mathrm{MB}_{12}$ with increased filling of the conduction band $\left(\mathrm{ZrB}_{12}, \mathrm{HfB}_{12}, \mathrm{UB}_{12}\right)$, comparatively to $\mathrm{M}^{3+} \mathrm{B}_{12}$ compounds. The total energy calculations for different magnetic configurations in $\mathrm{YbB}_{12}$ point to a possibility of antiferromagnetic coupling between $\mathrm{Yb}^{3+}$ ions.

PACS: 62.20.D- Elasticity;

71.15.Nc Total energy and cohesive energy calculations;

71.20.Lp Intermetallic compounds.
\end{abstract}

Keywords: rare-earth compounds, borides, bulk properties, electronic structure.

\section{Introduction}

The discovery of superconductivity (SC) in $\mathrm{MgB}_{2}$ at $T_{S C} \simeq 40 \mathrm{~K}$ has stimulated intensive investigations of boron-rich compounds. Among them, the $\mathrm{MB}_{6}$ hexaborides and $\mathrm{MB}_{12}$ dodecaborides ( $\mathrm{M}$ is alkaline-earth, rare-earth, early transition, or actinide metal) exhibit a wide range of peculiar physical properties [1-3], such as $\mathrm{SC}\left(\mathrm{YB}_{6}\right.$ and $\mathrm{ZrB}_{12}$, Refs. 1, 4-8), Kondo and valence fluctuation effects $\left(\mathrm{CeB}_{6}, \mathrm{SmB}_{6}\right.$, and $\mathrm{YbB}_{12}$, Refs. 9-11), colossal magnetoresistance $\left(\mathrm{EuB}_{6}[12-14]\right)$, and narrow gap semiconducting behavior $\left(\mathrm{YbB}_{6}[15,16]\right)$. Some hexaborides are expected to be candidates for thermoelectric con- version materials with very promising applications [17]. At the same time, there are scarce and conflicting published data on the elastic properties of hexaborides.

Also, ferromagnetism (FM) was recently reported at high temperatures in La doped alkaline-earth hexaboride compounds [18] (and later in $\mathrm{CaB}_{2} \mathrm{C}_{2}$ [19]), and the origin of this effect is still under discussion. The recent experimental and theoretical results related to anomalous FM of these borides and their electronic spectra are given in Refs. 20-25 and in the references therein. In addition, the trivalency of some fraction of $\mathrm{Yb}$ atoms and a related possibility of FM state in $\mathrm{YbB}_{6}$ have been reported [16]. Obviously, the magnetic semiconducting borides attract a 
considerable attention in the novel field of spintronics [20].

With the exception of $\mathrm{YbB}_{12}$, information on physical properties of the dodecaborides is still scarce. It was recently found, that heavy rare-earth (RE) dodecaborides demonstrate anomalous magnetic [26] and thermal $[27,28]$ properties. The dodecaborides are considered as hard and refractory materials, and these properties are related to peculiar chemical bonding in such compounds. However, little is known about elastic properties of $\mathrm{MB}_{12}$. In general, the $\mathrm{MB}_{6}$ and $\mathrm{MB}_{12}$ borides are of great scientific interest and technological importance due to their extraordinary electronic, magnetic, and structural properties. Remarkably, that compounds with comparatively simple cubic crystal structures possess such variety of physical properties.

In the present work we are focused on theoretical and experimental studies of electronic structure and bulk properties of two series of borides, $\mathrm{MB}_{6}(\mathrm{M}=\mathrm{Y}, \mathrm{Ca}, \mathrm{Sr}$, $\mathrm{Ba}, \mathrm{La}, \mathrm{Eu}, \mathrm{Gd}, \mathrm{Yb})$ and $\mathrm{MB}_{12}(\mathrm{M}=\mathrm{Y}, \mathrm{Zr}, \mathrm{Ho}, \mathrm{Er}, \mathrm{Tm}$, $\mathrm{Yb}, \mathrm{Lu}, \mathrm{Hf}, \mathrm{Th}, \mathrm{U})$. In order to shed light on the origin of the intriguing properties of these borides, the corresponding ab initio calculations of electronic structures are carried out within consistent and reliable computational techniques. In the experimental part, the high-precision measurements of the lattice parameters and elastic constants have been performed for $\mathrm{ZrB}_{12}, \mathrm{HoB}_{12}, \mathrm{ErB}_{12}$, $\mathrm{TmB}_{12}, \mathrm{LuB}_{12}, \mathrm{YB}_{6}$ and $\mathrm{LaB}_{6}$ borides. The main objective of these studies was to determine the principal electronic states and interactions responsible for the bulk properties of the representative compounds of $\mathrm{MB}_{6}$ and $\mathrm{MB}_{12}$ series. The effects of magnetic interactions, magnetic ordering, and superconductivity have been also addressed, when it was pertinent. Also, a comparison of the experimental data and the calculated volume dependent band structures is expected to be very useful for development of advanced theoretical models for electronic structure of rare-earth compounds.

\section{Experimental details}

The process of sample preparation consists of the synthesis of dodecaborides by a borothermal reduction of the metal oxides in vacuum at $1900 \mathrm{~K}$, the compacting of these powders into rods and their subsequent sintering, and of inductive zone melting. The purity of the initial oxides $\mathrm{M}_{2} \mathrm{O}_{3}$ was $4 \mathrm{~N}$. The content of the main substance in the initial amorphous boron was no less than $99.5 \%$. Highly volatile impurities in boron were deleted partially during the synthesis procedure and partially during zone melting. The total content of impurities in samples studied was not higher than $10^{-3}$ mass $\%$. The $\mathrm{YB}_{6}$ and $\mathrm{LaB}_{6}$ samples have been obtained in an analogous way.

The ultrasound studies were performed on samples which were cut from the zone-melted single crystals. The single crystals were grown using correspondingly oriented seeds. According to the x-ray topography and Laue photograph analysis, their real structures are highly perfect. Electron diffraction and high-resolution transmission microscopy analysis allowed us to determine the characteristics of local structural details of the single crystals: according to the electron Kikuchi patterns and point electron diffraction patterns, which correspond only to the $\mathrm{UB}_{12}$ or $\mathrm{CaB}_{6}$ type structures, defects were practically absent.

The sound velocity measurements in borides were performed at the liquid nitrogen temperature along the principal crystallographic axes by employing the phasefrequency method within the frequency range of $53-55 \mathrm{MHz}$. In this method the phase shift in a sample can be represented by

$$
\Phi=2 \pi f L / s=2 \pi n+\delta \varphi,
$$

where $f$ is frequency, $s$ is velocity of the sound, $L$ is thickness of a sample, $n \geq 0$ is integer number. For the studied samples $L \sim 3-10 \mathrm{~mm}$ and $s \sim(6-10) \cdot 10^{5} \mathrm{~cm} / \mathrm{s}$, i.e., the value of $n$ is estimated to be about $10-100$. In the first stage, the sound velocity was estimated from a slope of the phase-frequency characteristic of a sample, $d \Phi / d f=$ $=2 \pi L / \mathrm{s}$, measured by the difference method. This procedure permitted to eliminate the phase shifts introduced by piezotransducers and feeders. For $n>30$, corresponding to large $L$ and small $s$, such measurements allowed to obtain immediately the sound velocity with acceptable accuracy. In the opposite case, this first stage provided the exact value of $n$, and thereafter $s$ can be evaluated from $\delta \varphi$ value (see Ref. 29 for more details). By this means the accuracy of sound velocity measurements for the borides (Table 1) is estimated to be not worse than $0.5 \%$.

In order to obtain all main elastic constants in a cubic crystal, one needs to measure the velocities of three eigen modes as sound propagates along $C_{2}$ axis [30]. These modes are

$$
\begin{gathered}
C_{44}(\mathbf{q}\|[110], \mathbf{u}\|[001]), \\
\left(C_{11}-C_{12}\right) / 2(\mathbf{q} \|[110], \mathbf{u} \perp[001]), \\
C^{\prime}=\left(C_{11}+C_{12}\right) / 2+C_{44}(\mathbf{q}\|[110], \mathbf{u}\|[110]) .
\end{gathered}
$$

Here $\mathbf{q}$ is the wave vector, $\mathbf{u}$ is the polarization. Then the velocity $s_{4}$ of the $C_{11}$ mode can be evaluated as $s_{4, \text { calc }}=\sqrt{s_{2}^{2}+s_{3}^{2}-s_{1}^{2}}$. However, here we measured the velocity of the $C_{11}$ mode directly (q ||$[100]$, $\left.\mathbf{u}||[100]\right)$. This allowed to obtain more accurately values of the $C_{11}$ mode velocities and, in addition, to verify our measurements at $\mathbf{q} \|$ [110] by comparing measured and calculated values of $s_{4}$ (the last two columns in Table 1). It is clearly seen, that the discrepancies between these values are well within the accepted accuracy of our measurements. 
Table 1. Sound velocities in borides (in $10^{5} \mathrm{~cm} / \mathrm{s}$ ) measured at $T=78 \mathrm{~K}$.

\begin{tabular}{c|c|c|c|c|c}
\hline \multirow{2}{*}{ Compound } & \multicolumn{5}{|c}{ Sound velosities } \\
\cline { 2 - 6 } & $\begin{array}{c}s_{1} \|[110], \\
\mathbf{u} \|[001]\end{array}$ & $\begin{array}{c}s_{2} \\
\mathbf{q} \|[110], \\
\mathbf{u} \perp[001]\end{array}$ & $\begin{array}{c}s_{3} \\
\mathbf{q} \|[110], \\
\mathbf{u} \perp[110]\end{array}$ & \multicolumn{2}{|c}{\begin{tabular}{c}
\multicolumn{2}{|c}{$s_{4} \|[100]}$, \\
$\mathbf{u} \|[100]$
\end{tabular}} \\
\cline { 5 - 7 } & & & & exp. & calc. \\
\hline \hline $\mathrm{YB}_{6}$ & 3.11 & 7.47 & - & 10.77 & - \\
$\mathrm{LaB}_{6}$ & 4.35 & 6.6 & - & 9.9 & - \\
$\mathrm{ZrB} 12$ & 8.56 & 6.6 & 12.3 & 11.1 & 10.98 \\
$\mathrm{HoB} 12$ & 7.3 & 5.9 & 10.5 & 9.6 & 9.56 \\
$\mathrm{ErB} 12$ & 7.48 & 5.91 & 10.6 & 9.6 & 9.57 \\
$\mathrm{TmB} 12$ & 7.28 & 5.8 & 10.6 & 9.7 & 9.64 \\
$\mathrm{LuB} 12$ & 7.71 & 5.8 & 10.9 & 9.62 & 9.61 \\
\hline \hline
\end{tabular}

Table 2. Elastic constants of borides measured at $T=78 \mathrm{~K}$.

\begin{tabular}{c|c|c|c|c|c|c|c|c}
\hline \hline & \multicolumn{7}{|c}{ Elastic constants } \\
$\begin{array}{c}\text { Com- } \\
\text { pound }\end{array}$ & $\begin{array}{c}\rho, \\
\mathrm{g} / \mathrm{cm}^{3}\end{array}$ & $\begin{array}{c}C_{11}, \\
\mathrm{GPa}\end{array}$ & $\begin{array}{c}C_{44}, \\
\mathrm{GPa}\end{array}$ & $\begin{array}{c}C_{12}, \\
\mathrm{GPa}\end{array}$ & $\begin{array}{c}B, \\
\mathrm{GPa}\end{array}$ & $v$ & $A_{Z}$ & $\begin{array}{c}\Theta_{D} \\
\mathrm{~K}\end{array}$ \\
\hline \hline $\mathrm{YB}_{6}$ & 3.706 & 430 & 36 & 16 & 154 & 0.036 & 0.17 & $650,706[8]$ \\
$\mathrm{LaB}_{6}$ & 4.710 & 463 & 89 & 45 & 184 & 0.089 & 0.43 & 770 \\
$\mathrm{ZrB}_{12}$ & 3.612 & 443 & 265 & 129 & 234 & 0.226 & 1.69 & $1260,1000[5]$ \\
$\mathrm{HoB}_{12}$ & 4.655 & 429 & 248 & 105 & 213 & 0.197 & 1.53 & 1085 \\
$\mathrm{ErB}_{12}$ & 4.706 & 434 & 263 & 105 & 214 & 0.195 & 1.60 & 1100 \\
$\mathrm{TmB}_{12}$ & 4.756 & 448 & 252 & 128 & 233 & 0.222 & 1.58 & 1080 \\
$\mathrm{LuB}_{12}$ & 4.868 & 450 & 290 & 123 & 232 & 0.215 & 1.77 & $1110,1190[28]$ \\
\hline \hline
\end{tabular}

$B=\left(C_{11}+2 C_{12}\right) / 3$ is the bulk modulus, $v=C_{12} /\left(C_{11}+C_{12}\right)$ is the Poisson ratio, $A_{Z}=2 C_{44} /\left(C_{11}-C_{12}\right)$ is Zener's elastic anisotropy parameter, $\Theta_{D}$ is the Debye temperature (present work and specific heat data of Refs. $5,8,28)$, $\rho$ is the x-ray density.

The evaluated elastic constants, bulk moduli, and Debye temperatures of the borides are given in Table 2. The Debye temperatures were calculated according to the relationship (Ref. 30, in Gauss units):

$$
\Theta_{D}=0.0036265\left(\frac{\rho z}{A I}\right)^{1 / 3},
$$

where $\rho$ is the x-ray density, $A$ is the molecular weight, $z$ is the number of oscillators in the molecule (we assumed $z=13$ and $z=7$ for dodecaborides and hexaborides, respectively), $I$ is the sum of inverse cubes of the elastic waves velocities, averaged over all directions of the wave normal [30]. The obtained by this way values of $\Theta_{D}$ are in agreement with the results of specific heat studies in $\mathrm{ZrB}_{12}$ [5], $\mathrm{LuB}_{12}$ [28], and $\mathrm{YB}_{6}$ [6] (see Table 2). The measured lattice parameters are listed in Tables 3 and 4. These values appeared to be in accordance with the previously published data (see. Ref. 3 and references therein).

Table 3. Experimental and theoretical values of lattice constants and bulk moduli of $\mathrm{MB}_{6}$ hexaborides.

\begin{tabular}{c|c|c|c|c}
\hline \hline Compound & $a_{\text {exp }}, \AA$ & $a_{\text {theor }}, \AA$ & $B_{\text {exp }}, \mathrm{GPa}$ & $B_{\text {theor }}, \mathrm{GPa}$ \\
\hline $\mathrm{CaB}_{6}$ & $4.152[3]$ & 4.131 & - & 159 \\
$\mathrm{SrB}_{6}$ & $4.197[3]$ & 4.166 & - & 160 \\
$\mathrm{BaB}_{6}$ & $4.269[3]$ & 4.230 & - & 162 \\
$\mathrm{EuB}_{6}$ & 4.1845 & 4.164 & $167[13]$ & 159 \\
$\mathrm{YbB}_{6}$ & $4.1468[3]$ & 4.079 & $166[13]$ & 159 \\
$\mathrm{YB}_{6}$ & 4.1002 & 4.080 & $154,189[44]$ & 179 \\
$\mathrm{LaB}_{6}$ & 4.1565 & 4.130 & $184,170[13], 186[43]$ & 184 \\
$\mathrm{CdB}_{6}$ & $4.1066[3]$ & 4.112 & $170[43]$ & 175 \\
\hline \hline
\end{tabular}

Table 4. Experimental and theoretical values of lattice constants and bulk moduli of $\mathrm{MB}_{12}$ dodecaborides.

\begin{tabular}{c|c|c|c|c}
\hline \hline Compound & $a_{\text {exp }}, \AA$ & $a_{\text {theor }}, \AA$ & $B_{\text {exp }}, \mathrm{GPa}$ & $B_{\text {theor }}, \mathrm{GPa}$ \\
\hline $\mathrm{YB} 12$ & 7.500 & 7.45 & - & 224 \\
$\mathrm{ZrB} 12$ & 7.4077 & 7.331 & 234 & 249 \\
$\mathrm{HoB} 12$ & 7.4923 & 7.45 & 213 & 215 \\
$\mathrm{ErB}_{12}$ & 7.4841 & 7.443 & 214 & 217 \\
$\mathrm{TmB}_{12}$ & 7.4752 & 7.424 & 233 & 220 \\
$\mathrm{LuB} 12$ & 7.4644 & 7.413 & 232 & 223 \\
$\mathrm{HfB} 12$ & $7.377[3]$ & 7.349 & - & 243 \\
$\mathrm{ThB}_{12}$ & $7.612[3]$ & 7.543 & - & 239 \\
$\mathrm{UB} 12$ & $7.470[3]$ & 7.399 & $249[45]$ & 250 \\
\hline \hline
\end{tabular}

\section{Details of calculations}

The hexaborides crystallize in the $\mathrm{CaB}_{6}$-type cubic crystal structure which can be described in terms of the $\mathrm{CsCl}$ lattice, where $\mathrm{M}$ atoms occupy $\mathrm{Cs}$ sites, and the $\mathrm{B}_{6}$ octahedra are situated in the $\mathrm{Cl}$ sites. The basic structural elements of the cubic dodecaborides are stable cubooctahedral $\mathrm{B}_{12}$ clusters. The corresponding $\mathrm{UB}_{12}$-type structure is similar to the simple rock-salt lattice, where $\mathrm{M}$ atoms and $\mathrm{B}_{12}$ cubooctahedrons occupy the $\mathrm{Na}$ - and $\mathrm{Cl}-$ sites, respectively. 
The $a b$ initio electronic structure calculations were carried out for the paramagnetic (PM), ferromagnetic (FM), and antiferromagnetic (AFM) phases of $\mathrm{MB}_{6}$ and $\mathrm{MB}_{12}$ by using the density functional theory in the local spin density approximation (LSDA) and the generalized gradient approximation (GGA) in the framework of the relativistic full potential linear muffin-tin orbital (FP-LMTO) method [31-33]. In contrast to previous band-structure calculations for borides (see, e.g., Refs. 20, 25), which were mainly based upon atomic sphere approximation (ASA) techniques, in the present FP-LMTO method one can avoid extra shape approximations imposed on the charge density or potential. The main details of the FP-LMTO method employed in the present work are given in Refs. 31-33.

In RE compounds the localized strongly correlated $4 f$ electrons still present a challenge to the band theory $[34,35]$. The results of first-principles calculations together with a wealth of experimental data (including bulk and Fermi surface properties) indicate, that in order to describe the ground state of RE compounds, it is feasible to consider the $4 f$ states as semi-localized core states, in line with Refs. 34, 35. Actually, the standard RE model [34] is employed in this work in the limit of the large Hubbard repulsion $U$ within the $a b$ initio LSDA and GGA schemes for the exchange-correlation effects. In other words, the localized $4 f$ states of RE are treated as spinpolarized outer-core wave functions, contributing to the total spin density, and the spin occupation numbers were fixed by applying the Russel-Saunders coupling scheme to the $4 f$ shell, which was not allowed to hybridize with conduction electrons.

The band structures of $\mathrm{MB}_{6}$ and $\mathrm{MB}_{12}$ were calculated self-consistently for a number of lattice parameters close to experimental ones. The bulk moduli $B$ and the theoretical lattice parameters $a$ are evaluated according to Ref. 31 from the calculated total energies as functions of volume, i.e. from the corresponding equations of states (EOS) $E(V)$, and listed in Tables 3 and 4. For metallic $\mathrm{MB}_{6}$ and $\mathrm{MB}_{12}$ compounds the calculated total and partial densities of electronic states (DOS) at the Fermi level, $N\left(E_{F}\right)$, are given in Table 5 .

In addition, to investigate the chemical bonding in hexa- and dodecaborides, the balanced crystal orbital overlap populations (BCOOP) [33] have been calculated for a number of the borides within the FP-LMTO technique. The BCOOP is defined as the orbital population weighted density of states with a «balancing» denominator [33], and this method is similar to the well known COOP, which is the solid-state generalization of the quantum chemical quantity called overlap population. The original COOP appeared to be a basis set dependent quantity, and in the limit of nearly linear dependent basis set the COOP overestimates the antibonding character of electronic states (see Refs. 33 and references therein). However, the BCOOP method greatly suppresses the basis set dependence and treats the bonding and antibonding states on more or less equal footing, in a balanced way. The BCOOP is an energy resolved quantity which is positive for bonding states and negative for antibonding states. Therefore it allows to discriminate between bonding and antibonding contributions to the chemical bond.

Also, in order to shed light on the electronic structure of the mixed-valent $\mathrm{SmB}_{6}$ and $\mathrm{YbB}_{12}$ compounds within the LSDA + $U$ approach [36], the ab initio pseudo-potential VASP-PAW method has been employed in the present work (see Ref. 37 for details).

Table 5. Total and partial densities of electronic states at $E_{F}$ for $\mathrm{MB}_{6}$ and $\mathrm{MB}_{12}$ compounds.

\begin{tabular}{|c|c|c|c|c|c|c|c|c|c|}
\hline \multirow{3}{*}{$\begin{array}{c}\text { DOS } \\
\text { states } / \text { Ry } \cdot \text { f.u. }\end{array}$} & \multicolumn{9}{|c|}{ Compound } \\
\hline & \multicolumn{5}{|c|}{$\mathrm{MB}_{6}$} & \multicolumn{4}{|c|}{$\mathrm{MB}_{12}$} \\
\hline & Y & $\mathrm{La}$ & $\mathrm{Gd}$ & $\mathrm{Eu}(\mathrm{PM})$ & $\mathrm{Eu}(\mathrm{FM})$ & Y & $\mathrm{Zr}$ & $\mathrm{Lu}$ & $\mathrm{U}$ \\
\hline$M_{S}$ & 0.23 & 0.10 & 0.14 & 0.00 & 0.00 & 0.04 & 0.07 & 0.05 & 0.02 \\
\hline$M_{p}$ & 0.13 & 0.13 & 0.04 & 0.00 & 0.00 & 0.02 & 0.07 & 0.02 & 0.68 \\
\hline$M_{d}$ & 6.81 & 4.90 & 5.93 & 0.27 & 0.35 & 4.41 & 6.60 & 4.21 & 2.09 \\
\hline$M_{f}$ & 1.19 & 3.72 & 0.19 & 0.01 & 0.06 & 1.24 & 1.79 & 1.15 & 65.63 \\
\hline$B_{S}$ & 0.25 & 0.14 & 0.29 & 0.02 & 0.02 & 0.37 & 0.57 & 0.40 & 0.43 \\
\hline$B_{p}$ & 2.88 & 3.07 & 3.29 & 0.52 & 1.87 & 7.65 & 8.87 & 7.49 & 14.52 \\
\hline Total & 11.49 & 12.06 & 9.88 & 0.82 & 2.30 & 13.73 & 17.97 & 13.32 & 83.37 \\
\hline$N_{\exp }^{\gamma}\left(E_{F}\right)$ & $23.4[8]$ & $14.2[40]$ & - & - & - & $18.0[28]$ & $25.5[5]$ & $23.8[28]$ & $116[41]$ \\
\hline
\end{tabular}




\section{Results and discussion}

\section{$\mathrm{MB}_{6}$}

There is no agreement on the nature of FM in the La-doped $\mathrm{CaB}_{6}$ and $\mathrm{SrB}_{6}$ : either it is the FM phase of a dilute electron gas (see, e.g., Ref. 19), or it is related to the presumed excitonic state [38], or be caused by distortions and vacancies of whole $\mathrm{B}_{6}$ clusters [23]. At the same time, the features of band overlap, or the presence of a small energy gap, have important implications for the models proposed to explain the magnetic properties of these borides [20,22,38].

The total and partial densities of electronic states $N(E)$ for $\mathrm{CaB}_{6}$, calculated for experimental values of lattice constants and atomic positions, as well as the corresponding principal BCOOPs, are shown in Fig. 1. It should be pointed out, that we also obtained very similar DOS and BCOOP for the isovalent alkaline-earth hexaborides, $\mathrm{SrB}_{6}$ and $\mathrm{BaB}_{6}$. In Fig. 1 one can clearly see bonding valence states, which are separated from the antibonding states of the conduction band by a small energy gap. However, the calculated BCOOP and the valence charge density indicate, that the chemical bonding in $\mathrm{CaB}_{6}$ is more complex. Whereas the $\mathrm{B}-\mathrm{B}$ bonds are predominantly covalent, the $\mathrm{Ca}-\mathrm{B}$ bonds demonstrate a substantial ionic character.

Our calculations have also revealed, that for experimental values of lattice constants and atomic positions

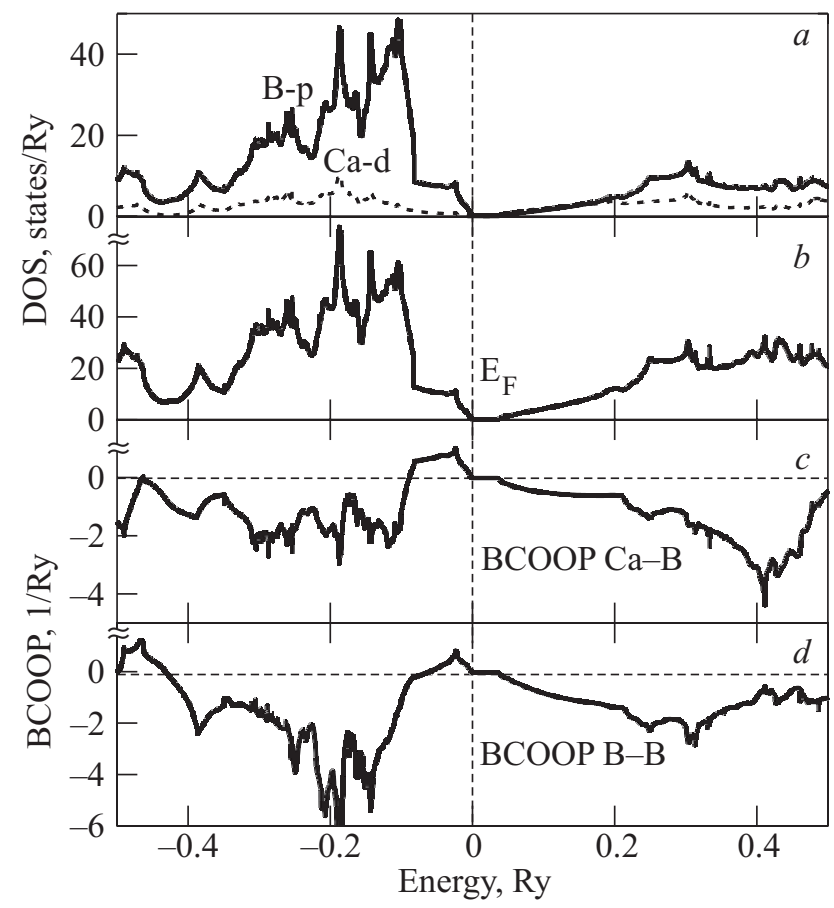

Fig. 1. Calculated densities of states and balanced crystal orbital overlap populations for $\mathrm{CaB}_{6}$ : $(a)$ partial boron $p$ (solid line) and calcium $d$ (dashed line) DOS; $(b)$ total DOS; $(c)$ Ca-B BCOOP; $(d)$ B-B BCOOP. the stoichiometric $\mathrm{CaB}_{6}, \mathrm{SrB}_{6}$, and $\mathrm{BaB}_{6}$ are semiconductors with small energy gaps: $0.48,0.16$, and $0.02 \mathrm{eV}$, correspondingly. These results are in a qualitative agreement with the recently obtained angle-resolved photoemission spectroscopy (ARPES) data [22], indicating that $\mathrm{CaB}_{6}$ is a semiconductor with the energy gap of about $1 \mathrm{eV}$. The difference between experiment and calculations would be expected, since the LDA calculations usually yield underestimated energy gaps. Previous band structure calculations, based on the LDA and ASA, provided semimetallic spectra for $\mathrm{CaB}_{6}, \mathrm{SrB}_{6}$, and $\mathrm{BaB}_{6}[20,25]$, whereas employment of the quasiparticle $G W$ approximation has given both semiconducting [20] and semimetallic [21] ground states for the divalent hexaborides. These contradictory results show that fine details of the band structure of hexaborides are very sensitive to specific implementations of $a b$ initio techniques.

Also, a value of the energy gap in alkaline-earth hexaborides is found to be related to the positional parameter $X$, which governs the difference between intra- and inter-octahedral B-B distances. These different B-B bond lengths become equal at $X=0.207$, and for this case the calculations provide the largest values of the energy gaps. Note, that experimental studies usually yield $X<0.207$, and the difference between calculated values of the energy gap, or even its absence, can be related to a chosen value of parameter $X$.

Though the $\mathrm{EuB}_{6}$ compound is also a divalent hexaboride, its transport and magnetic properties are complex and different from those of alkaline-earth hexaborides. The $\mathrm{EuB}_{6}$ orders partially ferromagnetically at $15 \mathrm{~K}$, then the resistivity and specific heat show another phase transition at $12.5 \mathrm{~K}$, with the majority of Eu spins become ferromagnetically aligned [14]. Here we did not address the origin of these phase transitions, as it lies beyond the aim of the present paper and capabilities of the methods employed. Instead, the detailed electronic structure calculations were carried out to shed light on the ground state of EuB 6 , and to provide a reliable basis for further analysis of its peculiar properties.

According to our calculations, $\mathrm{EuB}_{6}$ is found to be semimetallic in both paramagnetic and FM phases (see the corresponding $N\left(E_{F}\right)$ in Table 5), and the higher value of $N\left(E_{F}\right)$ for the FM phase appeared to be in conformity with the observed temperature behavior of resistivity $[13,14]$. Also, the total energy appeared to be minimal precisely for the FM phase. It should be noted, however, that the spin polarization of the $4 f$ states of $\mathrm{Eu}$ does not influence substantially the electronic structure of EuB $_{6}$, and the energy splitting between minority and majority bands is very small in the vicinity of $E_{F}$. As a result, the calculated magnetic moment of $\mathrm{EuB}_{6}, 7.03 \mu_{B}$, is completly due to the half-filled $4 f$ shell, and the contribution of conduction electrons to the moment is very small. 
For $\mathrm{YbB}_{6}$ hexaboride we have obtained the semiconducting ground state with a tiny energy gap of about $0.05 \mathrm{eV}$. The assumed $\mathrm{Yb}^{2+}$ state is in agreement with the estimations of Ref. 16 , which indicate that $2 \%$ of the $\mathrm{Yb}$ ions presumably adopt $\mathrm{Yb}^{3+}$ configuration, and the rest of $\mathrm{Yb}$ ions are divalent. According to our calculations, the trivalent $\mathrm{Yb}^{3+}$ state would correspond to substantially higher total energy for $\mathrm{YbB}_{6}$, and the origin of the recently reported weak FM in $\mathrm{YbB}_{6}[16]$ is not clear.

Among trivalent metallic hexaborides the nonmagnetic $\mathrm{YB}_{6}$ and $\mathrm{LaB}_{6}$ compounds are superconductors with transition temperatures of about 7 and $0.1 \mathrm{~K}$, respectively [1]. The calculated DOS (total and partial) and BCOOP for $\mathrm{YB}_{6}$ and $\mathrm{LaB}_{6}$ are shown in Figs. 2 and 3, respectively. One can see that main features of the $N(E)$ curves are similar to those calculated for the divalent $\mathrm{CaB}_{6}$ (Fig. 1). The occupied energy bands are formed by hybrid boron $2 s-2 p$ states, related to the inter- and intra-octahedral $\mathrm{B}-\mathrm{B}$ bonds. The highest fully occupied bands are formed by $p$ states responsible for the inter-octahedral interactions. The partially occupied band contains a substantial contribution from cation states and has also a large dispersion, which reflects the delocalized character of $p$ and $d$ states at the bottom of the conduction band. As a result, there are two fairly broad peaks in $N(E)$ of hexaborides, corresponding to bonding and antibonding states and arising due to hybridization of $d$ states of metal and $p$ states of boron in predominantly covalent B-B

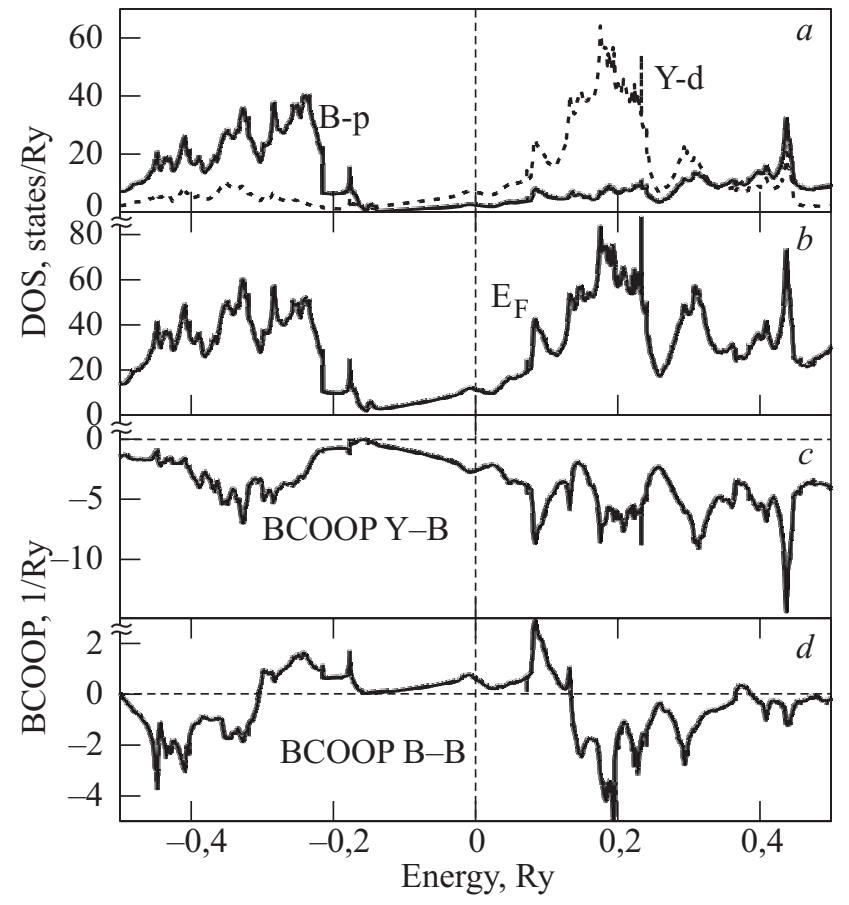

Fig. 2. Calculated densities of states and balanced crystal orbital overlap populations for $\mathrm{YB}_{6}$ : $(a)$ partial boron $p$ (solid line) and yttrium $d$ (dashed line) DOS; (b) total DOS; (c) Y-B BCOOP; $(d)$ B-B BCOOP.

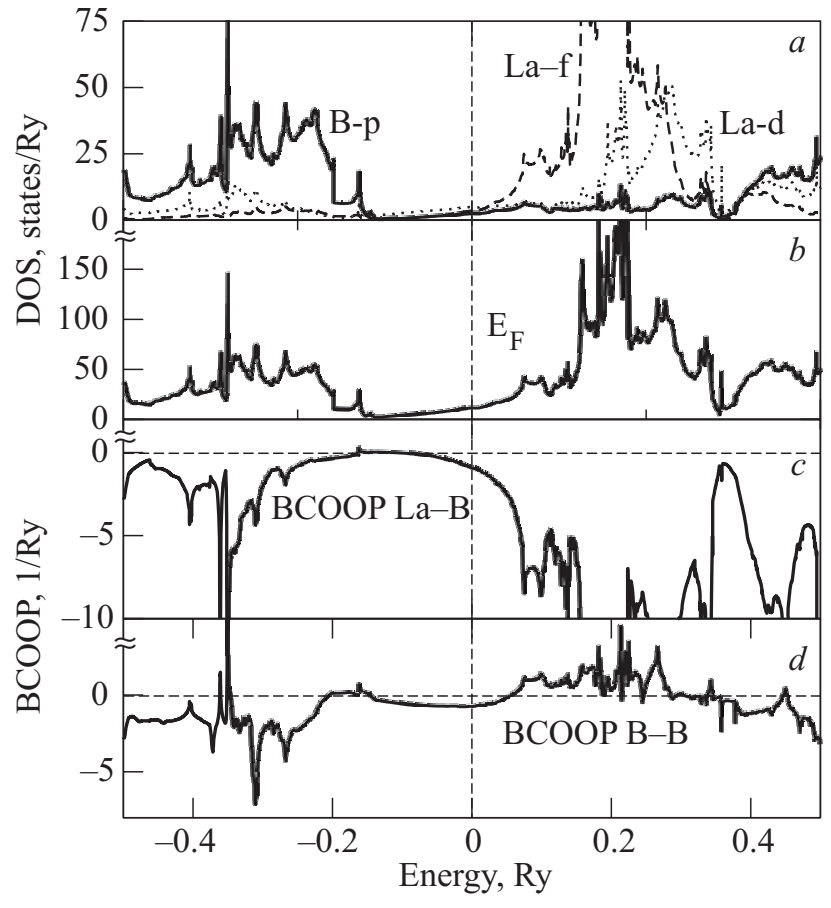

Fig. 3. Calculated densities of states and balanced crystal orbital overlap populations for $\mathrm{LaB}_{6}$ : $(a)$ partial boron $p$ (solid line), lanthanum $d$ (dotted line) and $f$ (dashed line) DOS; $(b)$ total DOS; (c) La-B BCOOP; (d) B-B BCOOP.

bonds inside and between $\mathrm{B}_{6}$ clusters. In general, the calculated band structure of $\mathrm{YB}_{6}$ is in agreement with the results of Ref. 39.

However, there are also notable distinctions between electronic structures of $\mathrm{YB}_{6}$ and $\mathrm{LaB}_{6}$. As can be seen in Fig. 3 and Table 5 , the $4 f$ states of La give a substantial contribution to DOS in $\mathrm{LaB}_{6}$ at the Fermi level, $N\left(E_{F}\right)$, and this provides the highest value of $N\left(E_{F}\right)$ among trivalent hexaborides. On the other hand, the aggregate of non- $f$-partial contributions to $N\left(E_{F}\right)$ is found to be higher in case of $\mathrm{YB}_{6}$ (see Table 5). Presumably, the sharp dissimilarity between $\mathrm{SC}$ transition temperatures of $\mathrm{YB}_{6}$ and $\mathrm{LaB}_{6}(7 \mathrm{~K}$ against $0.1 \mathrm{~K}$, respectively) can be related to a difference in symmetry of electronic states at $E_{F}$, and in the corresponding composition of partial contributions to $N\left(E_{F}\right)$. There is also a clearly seen «bump» in the $N(E)$ curve at $E_{F}$ for $\mathrm{YB}_{6}$ (see Fig. 2), whereas analogous feature is barely noticed in $N(E)$ for $\mathrm{LaB}_{6}$ in Fig. 3.

The calculated $N\left(E_{F}\right)$ can be compared with the corresponding «experimental» values taken from the measured electronic specific heat coefficients, $\gamma=2 \pi^{2} k_{B}^{2} N_{\exp }^{\gamma}\left(E_{F}\right) / 3$ (see Table 5). For this comparison, the calculated «bare» $N\left(E_{F}\right)$ have to be enhanced, presumably due to the electron-phonon interaction: $N_{\text {exp }}^{\gamma}\left(E_{F}\right)=(1+\lambda) N\left(E_{F}\right)$. This provides the estimations of many-body enhancement factors, $\lambda=1.0$ and $\lambda=0.2$ 
for $\mathrm{YB}_{6}$ and $\mathrm{LaB}_{6}$, respectively, which can contribute to the observed sharp difference in SC properties of these compounds. On the other hand, it was recently reported [42] that $\mathrm{YB}_{6}$ can not be definitively classified as either «exotic» or «conventional» superconductor, but instead this compound displays behavior which interpolates between the two regimes.

Another distinction between yttrium and lanthanum hexaborides resides in the chemical bonding. As one can see from the BCOOP plots in Fig. 2, the yttrium-boron bonding in $\mathrm{YB}_{6}$ is of an ionic character, whereas the $\mathrm{B}-\mathrm{B}$ bonding, as in the case of $\mathrm{CaB}_{6}$, has covalent features. This is also in agreement with the calculated charge density distribution for $\mathrm{YB}_{6}$, given in Fig. 4, where one can see no covalent bonds involving $\mathrm{Y}$ atoms. For the isoelectronic $\mathrm{LaB}_{6}$ the calculated BCOOP in Fig. 3 also demonstrates a clear picture of ionic bonding between $\mathrm{La}$ and $\mathrm{B}$ atoms, whereas there are no conspicuous covalent bonds between boron atoms. This suggests that the B-B bonding has some traits of metallic character, as well as weak La-La bonds, and therefore such bonding can be related to the high electron emissivity and low work function of $\mathrm{LaB}_{6}$.

As can be seen in Table 3, the calculated bulk moduli of $\mathrm{MB}_{6}$ are in a fair agreement with the available experimental data. It should be noted, however, that the listed in Table 3 bulk moduli of $\mathrm{EuB}_{6}(167 \mathrm{GPa})$ and $\mathrm{YB}_{6}$ (189 GPa) were estimated in Refs. 13 and 14 by interpolation, and these values should be used with great caution. Also, one can see in Table 3, that calculated values of bulk moduli for the trivalent hexaborides appeared to be about $10 \%$ larger than the theoretical $B$ of the divalent hexaborides. The Poisson ratio, $v=C_{12} /\left(C_{11}+C_{12}\right)$, is found to be very small for $\mathrm{LaB}_{6}$ and, especially, for $\mathrm{YB}_{6}$ (see Table 2) because of the large $C_{11}$ and the small $C_{12}$ values.

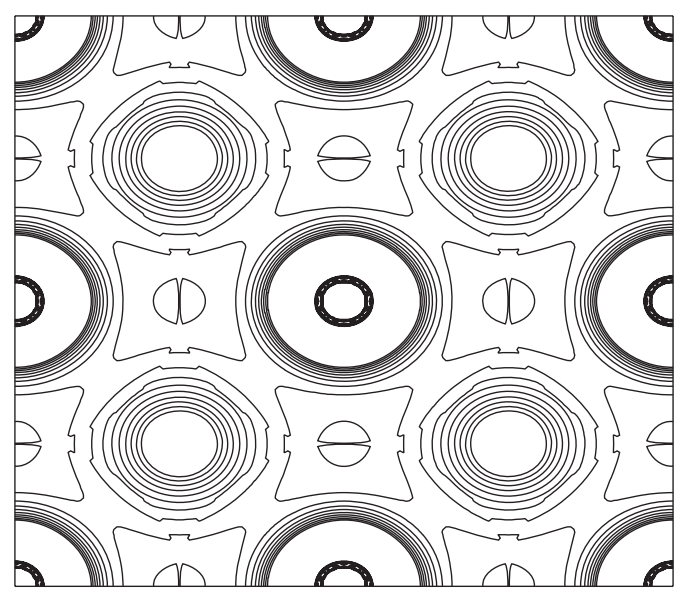

Fig. 4. Charge density for $\mathrm{YB}_{6}$ hexaboride in the plane crossing through the $\mathrm{Y}$ atoms. Sections through the boron bonds are also seen.
On the other hand, for the divalent hexaborides $\mathrm{EuB}_{6}$ and $\mathrm{YbB}_{6} v$ are expected to be higher, about 0.1 and 0.2 , respectively, due to larger values of $C_{12}$ (see Ref. 13). For all $\mathrm{MB}_{6}$ compounds the Cauchy relation, $C_{12}=C_{44}$, is not fulfilled, and this indicates noncentral force bonding, in accordance with the calculated BCOOPs. To characterize elastic anisotropy of a crystal, one usually employs Zener's anisotropy parameter, $A_{Z}=2 C_{44} /\left(C_{11}-C_{12}\right)$, which is close to 1 for an isotropic solid. As can be seen in Table $2, A_{Z}$ is substantially smaller than 1 in $\mathrm{YB}_{6}$ and $\mathrm{LaB}_{6}$, and this trend is expected to hold true for the whole $\mathrm{MB}_{6}$ series.

$$
\mathrm{MB}_{12}
$$

For the reference dodecaboride $\mathrm{YB}_{12}$ the calculated DOS and BCOOP are presented in Fig. 5. Very similar plots were obtained for the isovalent $\mathrm{LuB}_{12}$ compound. In general, the band structure of trivalent dodecaborides is determined by hybridization of the valence $s, p, d$ states of the metallic element with the $2 p$ states of boron. There are two groups of hybrid bands, separated by a «pseudogap» with substantial dispersion in the electronic spectrum at $E_{F}$. As is seen from the BCOOP plots in Fig. 5, the yttrium-boron bonding is of an ionic character, whereas the B-B bonding does not reveal a distinct picture of strong covalent bonds. Instead the $\mathrm{B}-\mathrm{B} \mathrm{BCOOP}$ is negative at low energies, then becomes positive, and at high energies it is negative again.

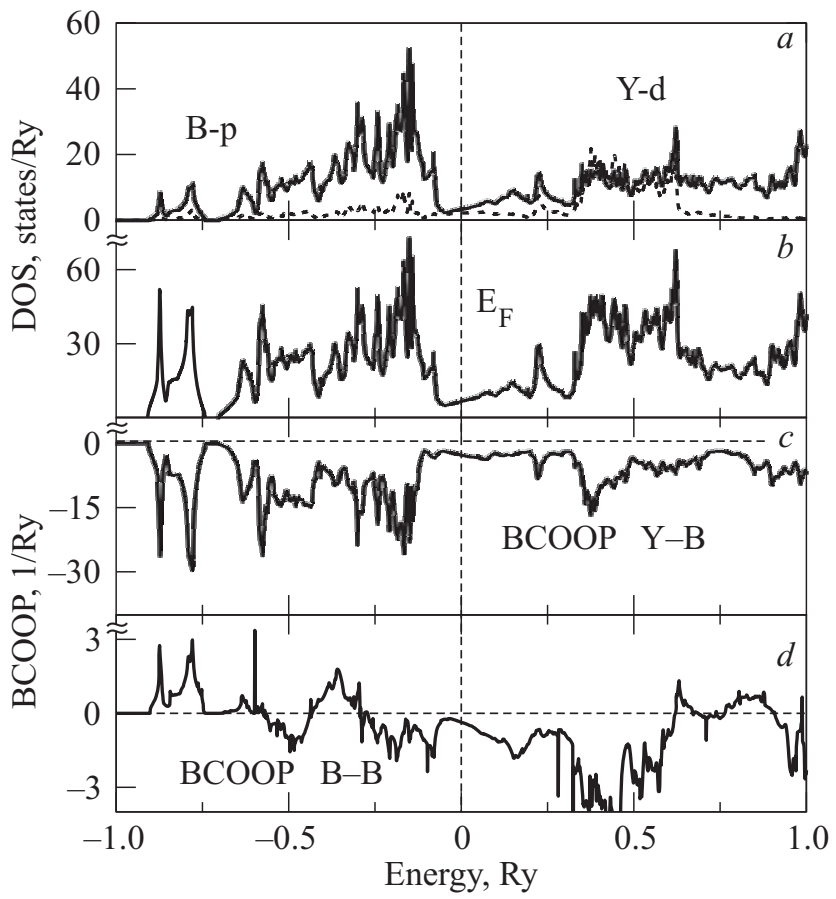

Fig. 5. Calculated densities of states and balanced crystal orbital overlap populations for $\mathrm{YB}_{12}$ : $(a)$ partial boron $p$ (solid line) and yttrium $d$ (dashed line) DOS; (b) total DOS; (c) Y-B BCOOP; $(d)$ B-B BCOOP. 


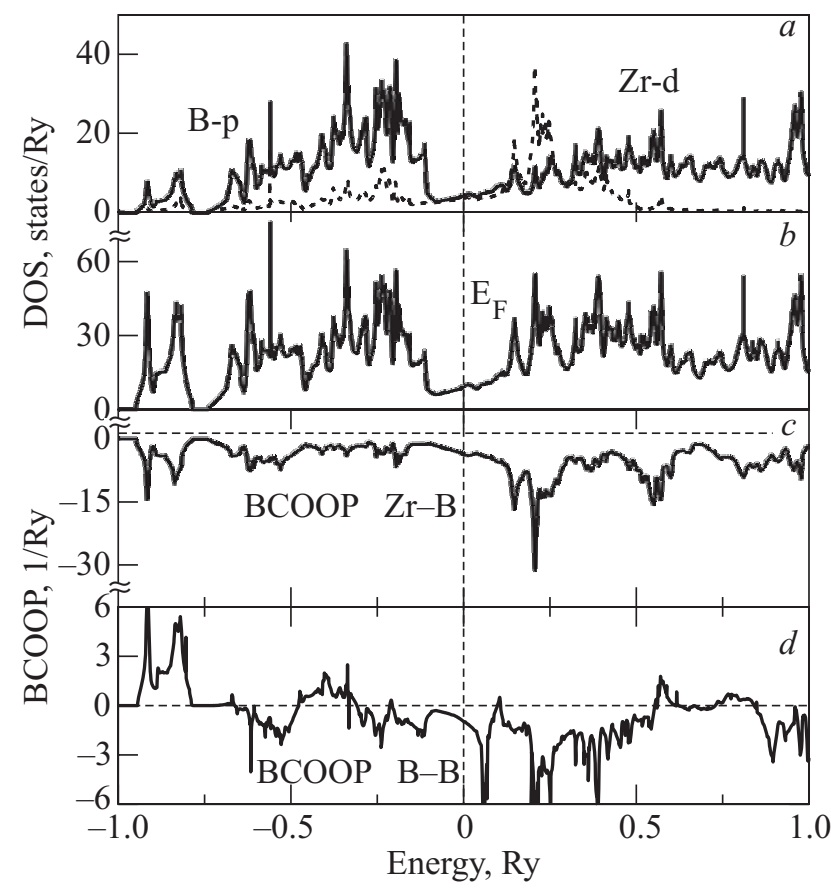

Fig. 6. Calculated densities of states and balanced crystal orbital overlap populations for $\mathrm{ZrB}_{12}$ : (a) partial boron $p$ (solid line) and zirconium $d$ (dashed line) DOS; $(b)$ total DOS; (c) $\mathrm{Zr}-\mathrm{B}$ BCOOP; (d) B-B BCOOP.

For the $\mathrm{ZrB}_{12}$ compound the calculated DOS and BCOOP in Fig. 6 are similar to the corresponding ones in $\mathrm{YB}_{12}$, and slight distinctions in their spectra are related to the larger band filling in $\mathrm{ZrB}_{12}$. The BCOOP in Fig. 6 also demonstrates the clear picture of ionic bonding between $\mathrm{Zr}$ and $\mathrm{B}$ atoms, whereas the bonds between boron atoms are complicated, covalent with, perhaps, small mixture of metallic character. This model of the bonding is consistent with the calculated charge density distribution for $\mathrm{ZrB}_{12}$ in Fig. 7.

In contrast to $\mathrm{YB}_{6}$, the $p$ electrons of boron provide the main contribution to the DOS of $\mathrm{YB}_{12}$ at $E_{F}$, whereas the contribution from $d$ electrons of yttrium appears to be comparatively small (see Fig. 5 and Table 5). On the other

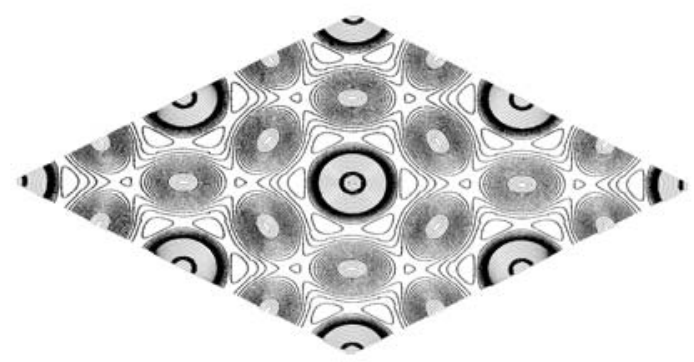

Fig. 7. Charge density for $\mathrm{ZrB}_{12}$ in the plane crossing through $\mathrm{Zr}$ atoms. Sections through the boron bonds are also seen. hand, in superconducting $\mathrm{ZrB}_{12}$ the partial contribution of $d$ electrons to $N\left(E_{F}\right)$ is found to be larger than the corresponding contributions in $\mathrm{YB}_{12}$ and $\mathrm{LuB}_{12}$. In Fig. 6 one can see a clear «bump» in $N(E)$ of $\mathrm{ZrB}_{12}$ at $E_{F}$, similar to the peak revealed in the DOS of superconducting $\mathrm{YB}_{6}$ (see Fig. 2).

The detailed analysis of SC in borides apparently goes beyond the aim of the present work, but it seems likely that substantial presence of $d$ electrons at $E_{F}$ is favorable for $\mathrm{SC}$ of $\mathrm{YB}_{6}$ and $\mathrm{ZrB}_{12}$. Also, estimated from the calculated $N\left(E_{F}\right)$ and experimental electronic specific heat data in Table 5, the electron-phonon coupling constants are found to be $\lambda=1.0$ and $\lambda=0.42$ in $\mathrm{YB}_{6}$ and $\mathrm{ZrB}_{12}$, respectively. This attracts attention to the origin of $\mathrm{SC}$ in $\mathrm{ZrB}_{12}$, which is a widely debated topic [4-7].

As can be seen in Table 5 , in $\mathrm{UB}_{12}$ the $5 f$ states of uranium yield a dominant contribution to DOS at $E_{F}$, providing the highest value of total $N\left(E_{F}\right)$ among dodecaborides. This value is in agreement with the experimental electronic specific heat data of Ref. 41, provided the electron-phonon coupling constant $\lambda=0.4$ is accepted. This confirms the itinerant $f$-electron picture for $\mathrm{UB}_{12}$, in which neither SC nor magnetic ordering was detected [41].

The theoretical and experimental values of the bulk moduli and the lattice parameters of $\mathrm{MB}_{12}$ are listed in Table 4. Taking into account the over-binding tendency of the LDA [31], which generally provides smaller lattice parameters and higher bulk moduli, one can see that the calculated $B$ and $a$ are in good agreement with the experimentally observed trends in the $\mathrm{MB}_{12}$ series. In particular, the lattice parameters decrease slightly in a linear fashion in the series of $\mathrm{HoB}_{12}, \mathrm{ErB}_{12}, \mathrm{TmB}_{12}$, and $\mathrm{LuB}_{12}$ due to the lanthanide contraction. It is also seen in Table 4, that the bulk moduli are expected to be higher for compounds which have more electrons in the conduction band, i.e. for $\mathrm{ZrB}_{12}, \mathrm{HfB}_{12}$, and $\mathrm{UB}_{12}$, as compared to $\mathrm{MB}_{12}$ with the trivalent (specifically RE) metals. For the dodecaborides the Poisson ratio $v$ appeared to be close to 0.2 (see Table 2), whereas $v \leq 0.1$ for the trivalent hexaborides. As to the elastic anisotropy parameter $A_{Z}$, it lies between 1.5 and 1.8 for the $\mathrm{MB}_{12}$ series as opposed to $A_{Z} \leq 0.5$ in $\mathrm{MB}_{6}$, though the deviations from the isotropic value $A_{Z} \simeq 1$ hold true in both series. As in the case of hexaborides, we note a departure from the Cauchy relation, $C_{12}=C_{44}$, which is attributed to the noncentral force bonding. This is in accordance with the calculated BCOOPs for $\mathrm{MB}_{12}$.

In the present study a particular attention has been given to the famous Kondo insulator $\mathrm{YbB}_{12}$, where the origin of the observed tiny energy gap (about $30 \mathrm{meV}$ ) remains controversial, both experimentally and theoretically [9-11,38]. In Ref. 9 a small hybridization gap was obtained for $\mathrm{YbB}_{12}$ within the LDA $+U$ approach assum- 


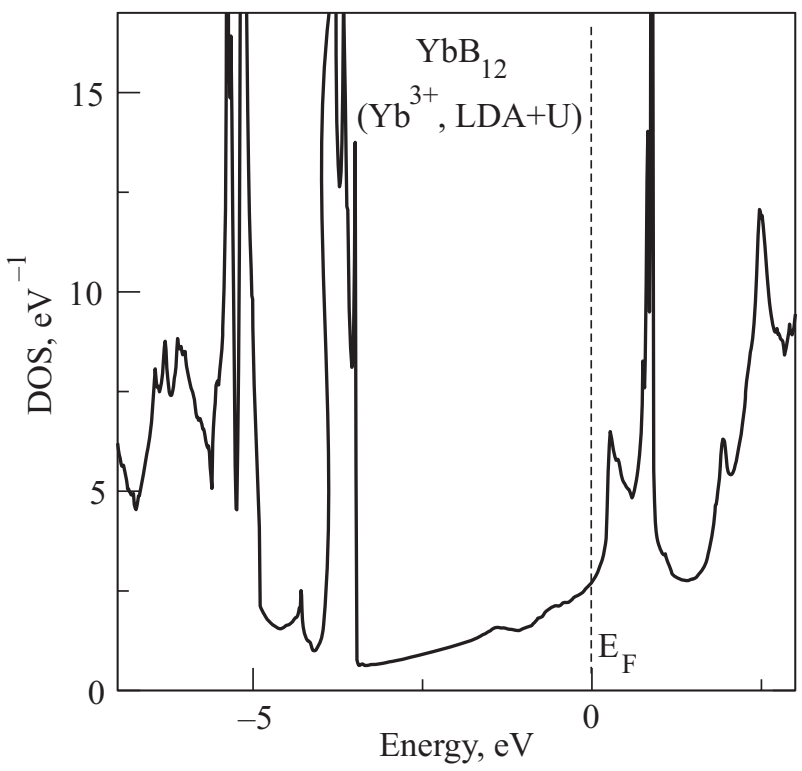

Fig. 8. Total density of states for $\mathrm{YbB}_{12}$ calculated within the LSDA $+U$ approach.

ing the divalent state of $\mathrm{Yb}$ ion. On the other hand, a wealth of experimental data indicate the valence state close to $\mathrm{Yb}^{3+}$ in $\mathrm{YbB}_{12}$ (see, e.g., Ref. 10 and references therein).

Here we tried to reach a true band theory limit in order to explain the experimental data and to explore a possibility of the hybridization gap in $\mathrm{YbB}_{12}$. For the $\mathrm{Yb}^{3+}$ state of ytterbium, we have employed the LSDA $+U$ approach in the framework of the VASP-PAW method [37]. The resulting DOS in Fig. 8 represents 13 hybridized $4 f$ energy bands below the Fermi energy $\left(E_{F}\right)$, and the 14th empty state just above $E_{F}$ with no hybridization gap. Even taking into consideration some uncertainty in deciding on a particular value of parameter $U$ ( 6 and $7 \mathrm{eV}$ were actually tried here), one can consider the obtained configuration in Fig. 8 as relevant to the formation of a Kondo singlet and compatible with an excitonic instability scenario of Ref. 38.

Obviously, our FP-LMTO LSDA and GGA calculations have not provided the energy gap for PM, FM, and AFM phases of $\mathrm{Yb}^{3+} \mathrm{B}_{12}$. However, for $\mathrm{YbB}_{12}$ the detailed calculations of the equation of state, $E(V)$, for different spin configurations point to a predominantly AFM coupling between $\mathrm{Yb}^{3+}$ ions. Specifically, the calculated ground state total energy for AFM configurations appeared to be about $20 \mathrm{meV}$ lower than the energy of the paramagnetic state. This is consistent with the recent neutron scattering spectrum studies for $\mathrm{YbB}_{12}[10,11]$, where the sharp $15 \mathrm{meV}$ peak in the low-energy peak structure has been attributed to $\mathrm{Yb}-\mathrm{Yb}$ AFM correlations in the PM state.

\section{Summary}

As a whole, the results of present electronic structure calculations for $\mathrm{MB}_{6}$ and $\mathrm{MB}_{12}$ are in agreement with the available experimental data on low temperature and bulk properties of higher borides. Our calculations confirm a semiconducting ground state of $\mathrm{CaB}_{6}, \mathrm{SrB}_{6}$, and $\mathrm{YbB}_{6}$ with the lower estimations for the energy gaps of $0.5,0.2$, and $0.05 \mathrm{eV}$, correspondingly, whereas a small overlap between conduction and valence bands is found in $\mathrm{EuB}_{6}$.

For metallic $\mathrm{R}^{3+} \mathrm{B}_{x}$ borides the calculated $\operatorname{DOS}\left(E_{F}\right)$ are in agreement with the electronic specific heat and $T_{S C}$ trends. In $\mathrm{YB}_{6}$ the electron-phonon interaction is estimated to be strong enough to provide the observed superconducting transition temperature of $7 \mathrm{~K}$. The itinerant $f$-electron picture is confirmed for $\mathrm{UB}_{12}$.

For the first time the elastic constants of a series of dodecaborides $\left(\mathrm{ZrB}_{12}, \mathrm{HoB}_{12}, \mathrm{ErB}_{12}, \mathrm{TmB}_{12}\right.$, and $\left.\mathrm{LuB}_{12}\right)$ and hexaborides $\left(\mathrm{YB}_{6}\right.$ and $\left.\mathrm{LaB}_{6}\right)$ were evaluated with one single experimental technique, and compared with the results of $a b$ initio calculations. The calculated bulk moduli are in agreement with the experimentally observed trends in the $\mathrm{MB}_{6}$ and $\mathrm{MB}_{12}$ series. In particular, the bulk moduli are expected to be higher for borides which possess more electrons in the conduction band.

Our calculations have shown that the bonding picture in the majority of $\mathrm{MB}_{6}$ and $\mathrm{MB}_{12}$ compounds can be considered as a mixture between covalent, metallic and ionic bonding. In particular, the BCOOP has been evaluated as a measure of the bonding character and a complementary quantity to the charge density analysis. Eventhough the BCOOP in the B-B case displays mostly covalent features, both for $\mathrm{MB}_{6}$ and $\mathrm{MB}_{12}$, it does not justify the consideration of these bonds as being purely covalent.

The calculated total energy for the AFM configuration of $\mathrm{YbB}_{12}$ is about $20 \mathrm{meV}$ lower than for the paramagnetic state. This is consistent with the neutron scattering spectrum studies for $\mathrm{YbB}_{12}$ by Alekseev et al. [10,11], where a sharp $15 \mathrm{meV}$ peak has been attributed to $\mathrm{Yb}-\mathrm{Yb}$ AFM correlations.

We are grateful to P.A. Alekseev, K. Flachbart, A.G. Grechnev, K. Siemensmeyer, N.E. Sluchanko, I.V. Svechkarev, and O.J. Żogal for fruitful scientific discussions.

This work has been supported by the Swedish Research Council (VR) and the Foundation for Strategic Research (SSF), by INTAS project 03-51-3036, and by the Russian-Ukrainian RFBR-NASU project 8-2008.

1. B.T. Matthias, T.H. Geballe, K. Andres, E. Corenzwit, G.W. Hull, and J.P. Maita, Science 159, 538 (1968).

2. J. Etourneau and P. Hagenmuller, Philos. Mag. B52, 589 (1985).

3. Handbook of High Temperature Compounds: Properties, Production, Applications, T.Ya. Kosolapova (ed.), Hemisphere Pub. Corp., New York (1990). 
4. M.I. Tsindlekht, G.I. Leviev, I. Asulin, A. Sharoni, O. Millo, I. Felner, Yu.B. Paderno, V.B. Filippov, and M.A. Belogolovskii, Phys. Rev. B69, 212508 (2004).

5. R. Lortz, Y. Wang, S. Abe, C. Meingast, Yu.B. Paderno, V. Filippov, and A. Junod, Phys. Rev. B72, 024547 (2005).

6. R. Khasanov, D. Di Castro, M. Belogolovskii, Yu. Paderno, V. Filippov, R. Brütsch, and H. Keller, Phys. Rev. B72, 224509 (2005).

7. V.A. Gasparov, N.S. Sidorov, and I.I. Zver'kova, Condmat/0508151 (2005).

8. R. Lortz, Y. Wang, U. Tutsch, S. Abe, C. Meingast, P. Popovich, W. Knafo, N. Shitsevalova, Yu.B. Paderno, and A. Junod, Phys. Rev. B73, 024512 (2006).

9. V.N. Antonov, B.N. Harmon, and A.N. Yaresko, Phys. Rev. B66, 165209 (2002).

10. P.A. Alekseev, J.-M. Mignot, K.S. Nemkovski, E.V. Nefeodova, N.Yu. Shitsevalova, Yu.B. Paderno, and R.I. Bewley, J. Phys.: Condens. Matter 16, 2631 (2004).

11. J.M. Mignot, P.A. Alekseev, K.S. Nemkovskii, L.P. Regnault, F. Iga, and T. Takabatake, Phys. Rev. Lett. 94, 247204 (2005).

12. J.L. Gavilano, B. Ambrosini, P. Vonlanthen, H.R. Ott, D.P. Young, and Z. Fisk, Phys. Rev. Lett. 81, 25 (1998).

13. S. Zherlitsyn, B. Wolf, B. Luthi, M. Lang, P. Hinze, E. Uhrig, W. Assmus, H.R. Ott, D.P. Young, and Z. Fisk, Eur. Phys. J. B22, 327 (2001).

14. J. Kunes and W.E. Pickett, Phys. Rev. B69, 165111 (2004).

15. J.M. Tarascon, J. Etourneau, P. Dordor, P. Hagenmuller, M. Kasaya, and J.M.D. Coey, J. Appl. Phys. 51, 574 (1980).

16. J.L. Gavilano, S. Mushkolaj, D. Rau, H.R. Ott, A. Bianchi, and Z. Fisk, Physica B329-333, 570 (2003).

17. M. Takeda, T. Fukuda, F. Domingo, and T. Miura, J. Solid State Chem. 177, 471 (2004).

18. D.P. Young, D. Hall, M.E. Torelli, Z. Fisk, J.L. Sarrao, J.D. Thompson, H.-R. Ott, S.B. Oseroff, R.G. Goodrich, and R. Zylser, Nature 397, 412 (1999).

19. J. Akimitsu, K. Takenawa, K. Suzuki, H. Harima, and Y. Kuramoto, Science 293, 1125 (2001).

20. H.J. Tromp, P. van Gelderen, P.J. Kelly, G. Brocks, and P.A. Bobbert, Phys. Rev. Lett. 87, 016401 (2001).

21. H. Kino, F. Aryasetiawan, K. Terakura, and T. Miyake, Phys. Rev. B66, 121103 (2002).

22. S. Souma, H. Komatsu, T. Takahashi, R. Kaji, T. Sasaki, Y. Yokoo, and J. Akimitsu, Phys. Rev. Lett. 90, 027202 (2003).

23. T. Jarlborg, J. Phys.: Condens. Matter 15, L249 (2003).

24. Z. Wu, D.J. Singh, and R.E.Cohen, Phys. Rev. B69, 193105 (2004).

25. S.-P. Gao, J. Jiang, M. Cao, J. Zhu, and J. Yuan, Phys. Rev. B69, 214419 (2004).
26. A. Kohout, I. Batko, A. Czopnik, K. Flachbart, S. Matas, M. Meissner, Y. Paderno, N. Shitsevalova, and K. Siemensmeyer, Phys. Rev. B70, 224416 (2004).

27. A. Czopnik, N. Shitsevalova, A. Krivchikov, V. Pluzhnikov, Yu. Paderno, and Y. Onuki, J. Solid State Chem. 177, 507 (2004).

28. A. Czopnik, N. Shitsevalova, V. Pluzhnikov, A. Krivchikov, Yu. B. Paderno, and Y. Onuki, J. Phys.: Condens. Matter 17, 5971 (2005).

29. E.A. Masalitin, V.D. Fil, K.R. Zhekov, A.N. Zholobenko, and T.V. Ignatova, Fiz. Nizk. Temp. 29, 93 (2003) [Low Temp. Phys. 29, 72 (2003)]; see also Cond-mat/0301183 (2003).

30. F.I. Fedorov, Theory of Elastic Waves in Crystal, Plenum Press, New York (1968).

31. O. Eriksson and J.M. Wills, in: Electronic Structure and Physical Properties of Solids, Hugues Dreysse (ed.), Springer, Berlin (2000).

32. G.E. Grechnev, R. Ahuja, and O. Eriksson, Phys. Rev. B68, 064414 (2003).

33. A. Grechnev, R. Ahuja, and O. Eriksson, J. Phys.: Condens. Matter 15, 7751 (2003); A. Grechnev, S. Li, R. Ahuja, O. Eriksson, U. Jansson, and O. Wilhelmsson, Appl. Phys. Lett. 85, 3071 (2004); P. Souvatzis, J.M. OsorioGuillen, R. Ahuja, A. Grechnev, and O. Eriksson, J. Phys.: Condens. Matter 16, 5241 (2004).

34. M.S.S. Brooks and B. Johansson, in: Ferromagnetic Materials, vol. 7, K.H.J. Buschow (ed.), North-Holland, Amsterdam (1993).

35. V.B. Pluzhnikov, A. Czopnik, G.E. Grechnev, N.V. Savchenko, and W. Suski, Phys. Rev. B59, 7893 (1999).

36. A.I. Liechtenstein, V.I. Anisimov, and J. Zaane, Phys. Rev. B52, R5467 (1995).

37. G. Kresse and D. Joubert, Phys. Rev. B59, 1758 (1999).

38. S. Curnoe and K.A. Kikoin, Phys. Rev. B61, 15714 (2000).

39. B. Jäger, S. Paluch, W. Wolf, P. Herzig, O.J. Żogal, N. Shitsevalova, and Y. Paderno, J. Alloys Compd. 383, 232 (2004).

40. E.F. Westrum, Jr., J.T.S. Andrews, B.H. Justice, and D.A. Johnson, J. Chem. Thermodynamics 34, 239 (2002).

41. M. Kasaya, F. Iga, K. Katoh, and T. Kasuya, J. Magn. Magn. Mater. 90-91, 521 (1990).

42. A.D. Hillier and R. Cywinski, Appl. Magn. Res. 13, 12 (1997).

43. S. Nakamura, T. Goto, S. Kunii, K. Iwashita, and A. Tamaki, J. Phys. Soc. Jpn. 63, 623 (1994).

44. H.E. King, S.J. La Placa, T. Penny, and Z. Fisk, in: Valence Fluctuations in Solids, L.M. Falikov, M.B. Maple, and W. Hanke (eds.), North-Holland, Amsterdam (1981).

45. J.-P. Dancausse, E. Gering, S. Heathman, and U. Benedict, J. Alloys Compd. 189, 205 (1992).

46. J. He, E. Wu, H. Wang, R. Liu, and Y. Tian, Phys. Rev. Lett. 94, 015504 (2005). 